Bull. Austral. Math. Soc.

VoL. 53 (1995) [267-269]

\title{
SET COVERING NUMBER FOR A FINITE SET
}

\section{H.-C. Chang and N. Prabhu}

Given a finite set $S$ of cardinality $N$, the minimum number of $j$-subsets of $S$ needed to cover all the $r$-subsets of $S$ is called the covering number $C(N, j, r)$. While Erdös and Hanani's conjecture that $\lim _{N \rightarrow \infty}(C(N, j, r)) /\left(\left(\begin{array}{l}N \\ r\end{array}\right) /\left(\begin{array}{l}j \\ r\end{array}\right)\right)=1$ was proved by Rödl, no nontrivial upper bound for $C(N, j, r)$ was known for finite $N$. In this note we obtain a nontrivial upper bound by showing that for finite $N$, $C(N, j, r) \leqslant\left(\left(\begin{array}{c}N \\ r\end{array}\right) /\left(\begin{array}{c}j \\ r\end{array}\right)\right) \ln \left(\begin{array}{c}N \\ r\end{array}\right)$.

Let $S$ be a set with $N$ elements. If $J_{1}, \ldots, J_{k}$ are $j$-subsets of $S$, (that is, subsets of $S$ of cardinality $j$ ) then $\mathcal{J}=\left\{J_{1}, \ldots, J_{k}\right\}$ is called a $k$-collection of $j$-subset of $S$. Further, the $k$-collection $\mathcal{J}$ is said to cover all the $r$-subsets of $S$, if for every $r$-subset $R$ of $S$, there is some $J_{i} \in \mathcal{J}, 1 \leqslant i \leqslant k$, such that $R \subset J_{i}$. The set covering number $C(N, j, r), j>r$, is the smallest integer $k$ such that some $k$-collection of $j$-subsets of $S$ covers all the $r$-subsets of $S$. Clearly

$$
C(N, j, r) \geqslant \frac{\left(\begin{array}{l}
N \\
r
\end{array}\right)}{\left(\begin{array}{l}
j \\
r
\end{array}\right)}
$$

and in 1963, Erdös and Hanani [1] conjectured that

$$
\lim _{N \rightarrow \infty} \frac{C(N, j, r)}{\left(\begin{array}{l}
N \\
r
\end{array}\right) /\left(\begin{array}{l}
j \\
r
\end{array}\right)}=1
$$

Erdös and Hanani's conjecture was proved by Rödl [2] in 1985. However, for finite $N$ a nontrivial upper bound for $C(N, j, r)$ was not known. In this note, using probabilistic arguments we obtain a nontrivial upper bound for $C(N, j, r)$ by proving:

Received 1 June 1995

Supported in part by NSF Research Initiation Award

Copyright Clearance Centre, Inc. Serial-fee code: $0004-9729 / 95 \$$ A2.00+0.00. 
Theorem 1. For $N \geqslant j>r$,

$$
C(N, j, r) \leqslant \frac{\left(\begin{array}{l}
N \\
r
\end{array}\right)}{\left(\begin{array}{l}
j \\
r
\end{array}\right)} \ln \left(\begin{array}{l}
N \\
r
\end{array}\right)
$$

Proof: Let $\mathrm{S}$ be a finite set of cardinality $N$. Let $\mathcal{J}=\left\{J_{1}, \ldots, J_{k}\right\}$ be a $k$-collection of $j$-subsets of $S$, where $J_{i}, 1 \leqslant i \leqslant k$ are chosen randomly and independently from among the $\left(\begin{array}{c}N \\ j\end{array}\right) j$-subsets of $S ; J_{1}, \ldots, J_{k}$ need not all be distinct.

Consider an $r$-subset $\mathrm{R}$ of $\mathrm{S}$. Then for any $j$-subset $\tilde{\mathrm{J}}$ of $\mathrm{S}$, the probability that $R \subset \tilde{\mathbf{J}}$ is

$$
\begin{aligned}
P[R \subset \overline{\mathrm{J}}] & =\frac{\left(\begin{array}{c}
N-r \\
j-r
\end{array}\right)}{\left(\begin{array}{l}
N \\
j
\end{array}\right)}=\frac{(N-r) !(N-j) ! j !}{N !(N-j) !(j-r) !}=\frac{(N-r) ! j ! r !}{N !(j-r) ! r !} \\
& =\frac{\left(\begin{array}{l}
j \\
r
\end{array}\right)}{\left(\begin{array}{l}
N \\
r
\end{array}\right)}
\end{aligned}
$$

Therefore

$$
P[R \not \subset \widetilde{\mathrm{J}}]=1-\frac{\left(\begin{array}{l}
j \\
r
\end{array}\right)}{\left(\begin{array}{l}
N \\
r
\end{array}\right)}
$$

The probability that none of the randomly chosen $j$-subsets of $\mathcal{J}$, namely $J_{1}, \ldots, J_{k}$, contains $R$ is hence

$$
R\left[\left(R \not \subset J_{1}\right) \wedge \ldots \wedge\left(R \not \subset J_{k}\right)\right]=\left(1-\frac{\left(\begin{array}{l}
j \\
r
\end{array}\right)}{\left(\begin{array}{l}
N \\
r
\end{array}\right)}\right)^{k}
$$

Label the $r$-subsets of $\mathrm{S}, R_{1}, \ldots, R_{\left(\begin{array}{c}N \\ r\end{array}\right)}$. Let $A_{i}$ be the event that $R_{i}$ does not belong to any of the $j$-subsets of $\mathcal{J}, 1 \leqslant i \leqslant\left(\begin{array}{c}N \\ r\end{array}\right)$. Then the probability that a randomly chosen $k$-collection of $j$-subsets $\mathcal{J}$ does not contain at least one $r$-subset is

$$
R\left[A_{1} \vee \ldots \vee A_{\left(\begin{array}{c}
N \\
r
\end{array}\right)}\right] \leqslant\left(\begin{array}{l}
N \\
r
\end{array}\right)\left(1-\frac{\left(\begin{array}{l}
j \\
r
\end{array}\right)}{\left(\begin{array}{l}
N \\
r
\end{array}\right)}\right)^{k}
$$


Therefore if $P\left[A_{1} \vee \ldots \vee A_{\left(\begin{array}{c}N \\ r\end{array}\right)}\right]<1$, then $P\left[\bar{A}_{1} \wedge \ldots \wedge \bar{A}_{\left(\begin{array}{c}N \\ r\end{array}\right)}\right]>0$ and hence some $k$-collection of $j$-subsets must cover all the $r$-subsets of S. $\bar{A}_{i}$ denotes the complement of the event $A_{i}, 1 \leqslant i \leqslant\left(\begin{array}{c}N \\ r\end{array}\right)$. Thus we want

$$
\left(\begin{array}{l}
N \\
r
\end{array}\right)\left(1-\frac{\left(\begin{array}{l}
j \\
r
\end{array}\right)}{\left(\begin{array}{l}
N \\
r
\end{array}\right)}\right)^{k}<1
$$

or

$$
k \ln \left(1-\frac{\left(\begin{array}{l}
j \\
r
\end{array}\right)}{\left(\begin{array}{l}
N \\
r
\end{array}\right)}\right)+\ln \left(\begin{array}{c}
N \\
r
\end{array}\right)<0
$$

or

$$
k>\frac{-\ln \left(\begin{array}{l}
N \\
r
\end{array}\right)}{\ln \left(1-\left(\begin{array}{l}
j \\
r
\end{array}\right) /\left(\begin{array}{l}
N \\
r
\end{array}\right)\right)} .
$$

The direction of the inequality is changed in equation (1) since, for $j<N,\left(\begin{array}{l}j \\ r\end{array}\right)<$ $\left(\begin{array}{c}N \\ r\end{array}\right)$ and hence $\ln \left(1-\frac{\left(\begin{array}{l}j \\ r\end{array}\right)}{\left(\begin{array}{l}N \\ r\end{array}\right)}\right)<0$.

For $0<x<1,-\ln (1-x) \geqslant x$, since $f(x)=e^{-x}-1+x \geqslant 0$ for $0<x<1$. $\left(f(0)=0\right.$ and $f^{1}(x)>0$ for $0<x<1$.) Hence

$$
k>\frac{\ln \left(\begin{array}{c}
N \\
r
\end{array}\right)}{\left(\begin{array}{l}
j \\
r
\end{array}\right) /\left(\begin{array}{l}
N \\
r
\end{array}\right)} \Rightarrow k>\frac{-\ln \left(\begin{array}{c}
N \\
r
\end{array}\right)}{\ln \left(1-\left(\begin{array}{l}
j \\
r
\end{array}\right) /\left(\begin{array}{l}
N \\
r
\end{array}\right)\right)} .
$$

Therefore, for any $k>\left(\left(\begin{array}{c}N \\ r\end{array}\right) /\left(\begin{array}{l}j \\ r\end{array}\right) \ln \left(\begin{array}{c}N \\ r\end{array}\right) i\right), P\left[\bar{A}_{1} \wedge \ldots \wedge \bar{A}_{\left(\begin{array}{c}N \\ r\end{array}\right)}\right]>0$ and hence there exists a $k$-collection of, $j$-subsets of $S$ which covers all the $r$-substs of $S$.

\section{REFERENCES}

[1] P. Erdös and H. Hanani, 'On a limit theorem in combinatorial analysis', Publ. Math. Debrecen 10 (1963), 10-13.

[2] V. Rödl, 'On a packing and covering problem', European J. Combin. 5 (1985), 69-78. 